Akademie der Landwirtschaftswissenschaften

der Deutschen Demokratischen Republik

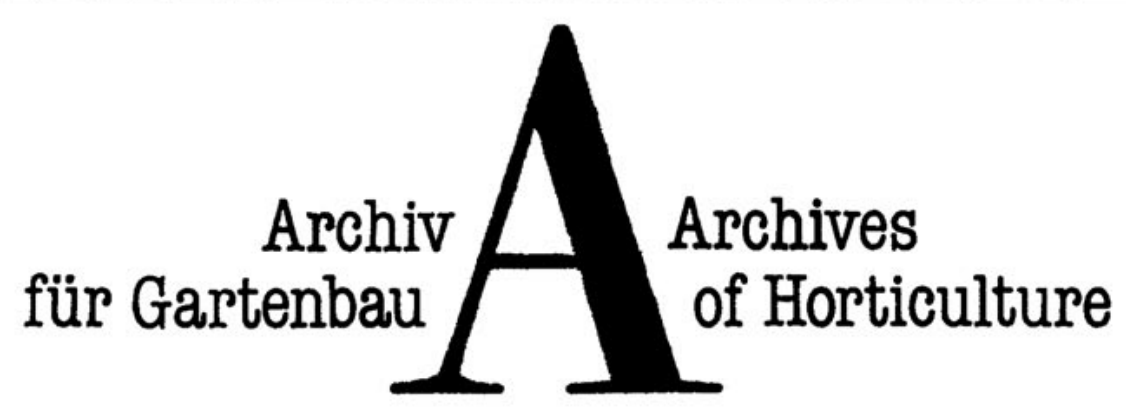

Volume $36 \cdot 1988 \cdot$ Number 7
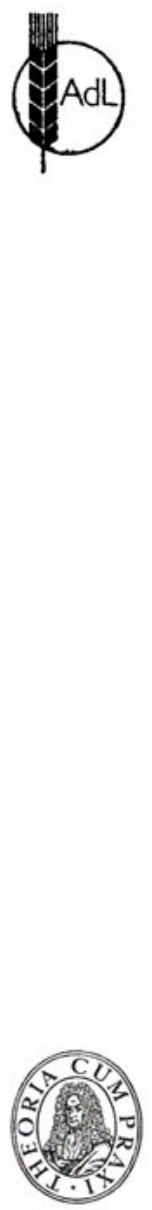

Akademie Verlag. Berlin

ISSN 0003-908X Aroh. Gartenbau, Berlin 36 (1988) 7, 395-450 


\section{Hinweise und Richtlinien für den Autor}

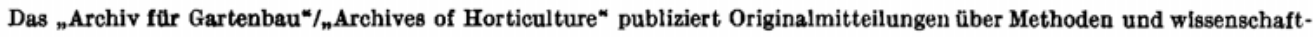
liche Ergebnisse des Obst-, Gemulse- und Zierpflanzenbaus.

Auf gedrăngte Darstellungsform ist zu achten. Eine Darstellung der Ergebnisse in mehreren Mitteilungen läBt sich nur ausnahmsweise befürworten. Umfangreiche Beiträge sollten als ,Informationsartikel erscheinen. Ihnen liegen ausführliche Beiträge zugrunde, die als Manuskripte in einem Fachdepot gespeichert und auf Wunsch eingesehen werden können. In der Regel werden nur abgeschlossene Untersuchungen veröffentlicht. Das schließt jedoch nicht aus, daB besonders bedeutungsvolle Teilergebnisse als kurze Mitteilung erscheinen. Ubersíchtsbeiträge können nur dann gedruckt werden, wenn sie eine straffe Verarbeitung des Stoffes erkennen lassen und von aktueller Bedeutung sind.

1.

Inhaltliche Gestaltung der Manuskripte

Die Einleitung ist 80 kurz wie möglich zu halten und hat sich nur auf die Problematik der Arbeit zu beschränken.

Nur die neueste und wichtigste Literatur ist heranzuziehen.

Uber Material und Methoden ist eine kurze Ubersicht, evtl. in Tabellenform, angebracht, sofern es sich nicht um neu entwickelte oder modifizierte Methoden handelt. Von einer Beschreibung bereits bekannter Verfahren ist unbedingt abzusehen. Die Darstellung dieses Abschnittes muB trotz aller Kürze dem Leser die genaue Beurteilung der Ergebnisse ermöglichen.

Die wichtigsten Ergebnisse sind in Form von übersichtlichen Tabellen oder graphischen Darstellungen mitzuteilen. Eine doppelte Darstellung als Tabelle und zugleich Abbildung ist unzulässig. Es ist zu vermeiden, die Angaben aus den Tabellen und Darstellungen in Worten nochmals in den Text einzubeziehen. Der Text in diesem Abschnitt hat sich nur auf das zum Verständnis der ausgeführten Ergebnisse sowie der daraus herzuleitenden Zusammenhănge Notwendige zu beziehen.

Die Diskussion der Ergebn is se muß auf der neuesten einschlägigen Literatur beruhen und zu klaren SchluBfolgerungen führen. Hinweise auf die weitere Kintwicklung der Arbeit sowie Schlubfolgerungen für Forschung und Praxis sind erwünscht, sie erhōhen den Informationswert des Beitrages. Keine Wiederholung zum vorhergehenden Abschnitt.

Die Zusammenfassung hat sich auf die Wiedergabe der wichtigsten Ergebnisse mit den daraus gezogenen Schlußfolgerungen zu beschränken und ist so zu formulieren, daB sie als Referat in Dokumentationsorgane übernommen werden kann.

2.

Technische Gestaltung der Manuskripte und Veröffentlichungen

Die Manuskripte sind zweifach an die Redaktion, Archiv für Gartenbau, Chefredakteur Prof. Dr. sc. FeHRMANN, Pillnitzer Platz 2, DDR - 8057 Dresden, zu senden.

Die Veröffentlichung russisch- und englischsprachiger Originalbeiträge ist möglich.

Die Schriftleitung nimmt nur Manuskripte an, deren Gesamtumfang 15 Schreibmaschinenseiten, einschl. Tabellen, Abbildungen und Literaturverzeichnis, nicht überschreitet (Manuskriptgestaltung nach TGL 6710).

Die Arbeit darf nicht, in anderer Form, im In- oder Ausland veröffentlicht worden sein.

Der Kopf des Manuskripts enthält in der gensnnten Reihenfolge:

Entstehungsort (Institution)

Vor- und Zuname des Autors (der Autoren)

Titel der Arbeit.

Für die Gliederung des Textes gilt TGL 0-1421.

Die Zusammenfassung (nicht länger als 20 Zeilen) ist in deutscher und möglichst auch in russischer und englischer Sprache zur Verfügung zu stellen. Gegebenenfalls wird die Ubersetzung von der Redaktion veranlaBt.

Das Literaturverzeichnis ist unter der Uberschrift „Literatu“ alphabetisch zu ordnen. Für die Zitierweise gelten TGL 20972 und TGL 20969.

Die Abbildungen sollen reproduktionsfăhig sein, d.h. Photos sind möglichst als Hochglanzabzüge zu liefern und zeichnerische Darstellungen mit schwarzer Tusche auf weißem Grund auszuführen (8. TGL 24470). Alle Abbildungen sind fortlaufend zu numerieren. Die Abbildungsunterschriften sind als gesondertes Verzeichnis beizufügen. Abbildungen sind nicht auf Text- oder Textrückseiten zu kleben.

Die Tabellen sind mit Uberschriften zu versehen und gesondert fortlaufend zu numerieren.

Im Text des Manuskriptes ist zu vermerken, wo die Abbildungen und Tabellen einzuordnen sind.

Formelzeichen sind nach TGL 0-1304, mathematische Zeichen nach TGL 0-1302 zu verwenden. Bei Maßen sind die gesetzlich vorgeschriebenen physikalisch-technischen Einheiten zu benutzen (Gesetzblatt der DDR, Sonderdruck Nr. 605 . 1. März 1969 - Anordnung über die Tafel der gesetzlichen Einheiten vom 26. Nov. 1968)

Am SchluB des Beitrages ist die Anschrift des Verfassers anzugeben.

Die Autoren erhalten Umbruchabzuge zur Korrektur (Korrekturzeichen nach TGL 0-16511) mit befristeter Termin. stellung. Bei Nichteinhaltung der Termine erteilt die Redaktion Imprimatur.

Alle TGL sind zu beziehen durch das Buchhaus Leipzig, Abteilung Standards, Postfach 140, DDR - 7010 Leipzig. 


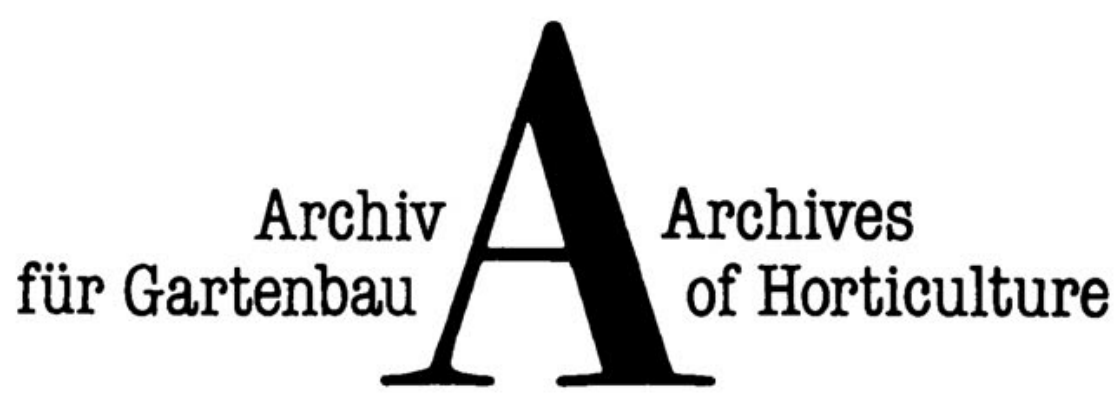

\section{Herausgeber}

Akademie der Landwirtschaftswissenschaften der DDR

\section{Chefredakteur}

Wolfgang Fehrmann, Dresden-Pillnitz

\section{Redaktionskollegium}

W. Blasse, Marquardt; H. Bochow, Berlin; H. Fröhlich, Großbeeren; F. Göhler, Großbeeren; F. Kaufmann, Berlin; H.-G. Kaufmann, Berlin;

H. Kegler, Aschersleben; F. Lenz, Bonn; A. Nisen, Gembloux;

J. Rumpel, Skierniewice; H. Rupprecht, Berlin; G. Stolle, Halle;

H.-J. Tantau, Hannover; G. J. Tarakanow, Moskau; G. Vogel, Großbeeren;

R. WeIChOLD, Quedlinburg; S. J. WeRthenM, Wilhelminadorp;

S. W. ZagaJa, Skierniewice; H. Znmmermann, Nossen

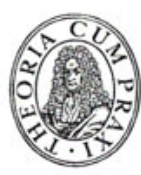


Das „Archiv für Gartenbau“/,Archives of Horticulture“ berichtet über Methoden, Untersuchungen und Ergebnisse aus Forschungs- und Entwicklungseinrichtungen sowie Betrieben der Obst-, Gemüse- und Zierpflanzenproduktion und verwandter Gebiete. Es werden biologische, acker- und pflanzenbauliche, technologische, technische und ökonomische Forschungsergebnisse mitgeteilt. Das Archiv informiert in Kurzbeiträgen über bedentsame neue wissenschaftliche Ergebnisse aus internationalen Schriften und üher Neverscheinungen einschlägiger wissenschaftlicher Standardwerke.

Bestellungen sind zu ricliten

- in der DDR an den Postzeitungsvertrieb unter Angabe der Kundennummer des Bestellenden oder an den AKADEMIE-VERLAG BERLIN, Leipziger StraBe 3-4, PF·Nr. 1233, DDR - 1086 Berlin;

- im sozialistischen Ausland an eine Buchhandlung für fremdsprachige Literatur oder an den zuständigen Postzeitungsvertrieb;

- in der BND und Berlin (West) an ejne Buchhandlung oder an die Auslieferungsstelle KUXST UNI WISSEN, Erich Bieber oHG, Postfach 102844, D - 7000 Stuttgart 10;

- in den übrigen vesteuropäischen Ländern an eine Buchhandlung oder an die Auslieferungsstelle KUNST UXD WISSEN, Erich Bieber GmbH, General Wille-Str. 4, CH - 8002 Zürich;

- im übrigen A usland an den Internationalen Buch- und Zeitschriftenhandel; den Buchexport, Volkseigener AuBenhandelsbetrieb der Deutschen Demokratischen Republik, Post fach 160, DDR - 7010 Leipzig, oder an den

AKADEMIE-VERLAf; BERLIN, Leipziger StraBe 3-4, PF-Nr. 1233, DDR - 1086 Berlin.

Zeitschrift „Archiv für Gartenbau“/,Archives of Horticul ture“

Herausgeber: Akademie der Landwirtschaftswissenschaften

der Deutschen Demokratischen Republik

Krausenstraße 38/39, DDR - 1086 Berlin.

Verlag: A kademie-Verlag Berlin, Leipziger Straße 3-4, PF-Nr. 1233, DDR - 1086 Berlin;

Fernruf: 2236201 oder 2236229 , Telex-Nr.: 114420 ;

Bank: Staatsbank der DDR, Berlin, Kto.-Nr.: 68 36-26-20־ 12.

Chefredakteur: Prof. Dr. sc. Wolfgaxg Fehryasi, Institut für Obstforschung Drezden-Pillnitz der AdL, Pillnitzer Platz 2, DDR - 8057 Dresden.

Anschrift der Redaktion: Institut für Obstforschung Dresden-Pillnitz der AdL, „Archiv für Gartenbau“, Pillnitzer Platz 2, DDR - 8057 Dresden.

Veröffentlicht unter der Lizenznummer 1276 des Presseamtes beim Vorsitzenden des Ministerrates der Deutschen Demokratischen Republik

Gesamtherstellung: VEB Druckerei „Gottfried Wilhelm Leibniz“, DDR - 4450 Gräfenhainichen.

Erscheinungsweise: Die Zeitschrift „Archiv für Gartenbau“/„Archives of Horticulture“ erscheint jăhrlich in einem Band mit 8 Heften. Das letzte Heft eines Bandes enthălt Inhalts-, Autoren- und Sachverzeichnis. Bezugspreis eines Bandes 240,- DM zuzüglich Versandspesen. Preis je Heft 30,- DM. Der gült ige Jahresbezugspreis für die DDR ist der Postzeitungsliste zu entnehmen.

Bestellnummer dieses Heftes: 1039/36/7.

Urheberrecht: Die Rechte über die in dieser Zeitschrift abgedruckten Arbeiten gehen ausschlieBlich an die Akademie der Landwirtschaftswissenschaften der Deutschen Demokratischen Republik über. Ein Nachdruck in anderen Zeitschriften oder eine Ubersetzung in andere Sprachen bedarf der Genehmigung der Akademie, ausgenonmen davon bleibt der Abdruck von Zusammenfassungen. Kein anderer Teil dieser Zeitschrift darf in irgendeiner Form - durch Photokopie, Mikrofilm oder ein anderes Verfahren - ohne schriftliche Genehmigung der Akademie reproduziert werden.

All rights reserved (including those of translation into foreign languages). No part of this issue, except the summaries may be reproduced in any form, by photoprint, microfilm or any other means, without written permission from the publishers.

(C) 1988 by Akademie-Verlag Berlin - Printed in the German Democratic Republic.

AN (EDV) 48236

00500 